\title{
THE MANY FACES OF LORENZ KNOTS
}

\author{
Marco AbATE \\ Dipartimento di Matematica \\ Università di Pisa \\ Largo Pontecorvo 5 \\ 56127 Pisa \\ Italia \\ Telefono: $+39 / 050 / 2213.230$ \\ Fax: $+39 / 050 / 2213.224$ \\ E-mail: abate@dm.unipi.it \\ Talk given in the Congress \\ "Matematica e Cultura 2011" \\ on March 26, 2011
}


One of the greatest pleasures in doing mathematics (and one of the surest signs of being onto something really relevant) is discovering that two apparently completely unrelated objects actually are one and the same thing. This is what Étienne Ghys, of the École Normale Superieure de Lyon, did a few years ago (see [1] for the technical details), showing that the class of Lorenz knots, pertaining to the theory of chaotic dynamical systems and ordinary differential equations, and the class of modular knots, pertaining to the theory of 2-dimensional lattices and number theory, coincide. In this short note we shall try to explain what Lorenz and modular knots are, and to give a hint of why they are the same. See also [2] for a more detailed but still accessible presentation, containing the beautiful pictures and animations prepared by Jos Leys [3], a digital artist, to illustrate Ghys' results.

\section{What is a knot?}

Informally speaking, a knot is a closed piece of string in space. More formally, a knot is a (globally injective) embedding of the circumference $S^{1}$ in the Euclidean 3-space $\mathbf{R}^{3}$. Two knots are considered the same if there is a way of continuously deform the space $\mathbf{R}^{3}$ so to bring the first knot exactly onto the second knot (or its mirror image). In more technical terms, two knots are equivalent if there is a homeomorphism of $\mathbf{R}^{3}$ (a bijective continuous transformation of the space onto itself with a continuous inverse) transforming the first knot in the second. In particular, a knot equivalent to the standard unit circumference in the plane is actually unknotted, and thus considered a trivial knot. See, e.g., [4] for a not exceedingly technical introduction to the mathematical theory of knots.

Mathematicians are entomologists at heart; they are prone to uncontrollable classification urges. For instance, one would like to have a list of all possible knots (up to equivalence, of course). The usual way for representing a knot consists in projecting it onto a plane so that the projection crosses itself in a finite number of points, and only two strands of knot pass through any crossing point. So one may look for the projection with the least number of self-crossings of a given knot (or, more precisely, of all equivalent forms of a given knot), and try to organize the knots according to this least number of self-intersections. For instance, the trivial knot clearly admits a representation with no self-crossings: the standard circumference. It is not difficult to see that knots admitting representations with only one or two self-crossings are actually unknots; so the first non-trivial knot is the trefoil knot, whose representation (see knot $3_{1}$ in Fig. 1) has exactly three self-crossings. Fig. 1 contains representations of distinct knots with at most 9 self-crossings.

A particular subclass of knots will be mentioned later on. A torus is a doughnut-shaped surface, that is the Cartesian product of two circumferences; a torus knot is a knot on a torus. In other words, 
in a torus knot the string winds on the surface of a torus. Fig. 2 contains the representations of the simplest torus knots; see [5] for more pictures of knots.

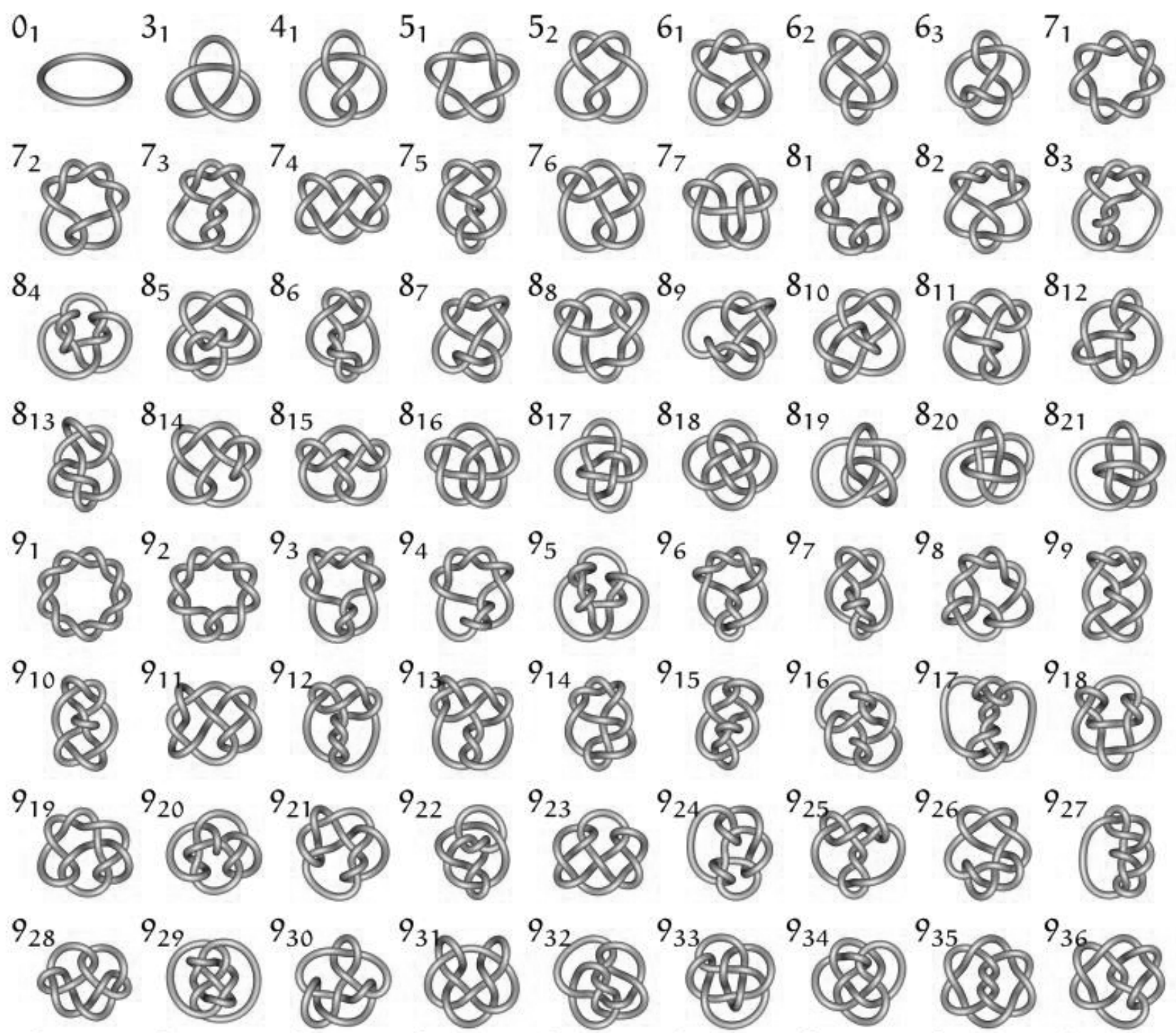

Fig. 1 Knots (from www.knotplot.com) 


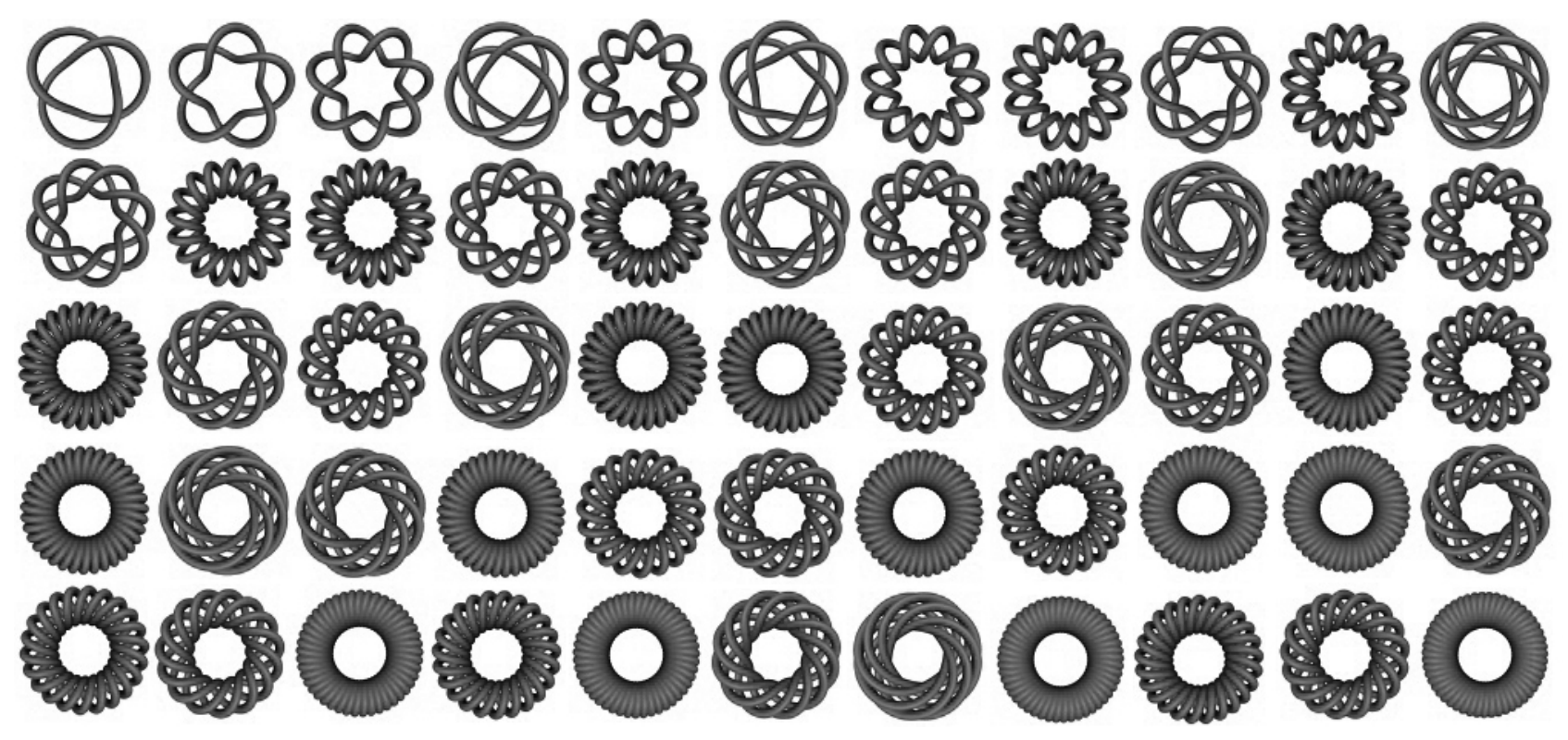

Fig. 2 Torus knots (adapted from www.knotplot.com)

\section{What is a Lorenz knot?}

Lorenz knots appear in the first, and still most famous, example of chaotic dynamical system, introduced by Edward N. Lorenz in 1963 [6] as a simplified model for convection in the atmosphere. This model consists of three (mildly non linear) ordinary differential equations:

$$
x^{\prime}=10(y-x), y^{\prime}=x(28-z)-y, z^{\prime}=x y-(8 / 3) z .
$$

How Lorenz noticed the presence of chaos in this system is by now almost legendary. He was solving numerically this system on a (large, for the time) computer, but he had to interrupt the computations for the night. The next day he gave as input to the computer the results of the computations of the previous afternoon, and soon noticed that the results he was obtaining were sensibly different from the ones he got the day before, even though the initial conditions were the same. Or were they? After several weeks of careful checking of the programs and computers involved to rule out any possible mistake, Lorenz realized that the data he entered the second day were only approximations of the data stored into the computer; and even though they were very good approximations (to the sixth decimal digit or so), this apparently negligible difference at the beginning provoked hugely different outcomes at the end.

Lorenz had discovered one of the most distinctive characteristics of chaotic dynamical systems: sensitive dependence on initial conditions. The slightest change in the initial state can cause a completely different result, the so-called (and by now exceedingly famous) butterfly effect. But in his model Lorenz also discovered another butterfly, which is more relevant to the present discussion. 
The Lorenz model, as any system of ordinary differential equations in three variables, prescribes at each point in space a velocity vector; we can then start from any point in space, and move according to the speed and direction given by these velocity vectors. The itinerary we follow is an orbit of the model. Lorenz noticed that almost all orbits tended to accumulate onto a peculiar and approximately butterfly-shaped set, having a very intricate geometric structure (later on it was proved that it is a fractal set of dimension slightly larger than two). This set, the Lorenz attractor, was the first example of strange attractor for a chaotic dynamical system; check [3] for beautiful pictures of the Lorenz attractor, and [7] to play with different orbits and see in real time how they accumulate onto the Lorenz attractor (and how they depend on the choice of initial conditions).

Most orbits go around wildly getting closer and closer to the Lorenz attractor; but a few special ones actually lives in the Lorenz attractor itself. These are the periodic orbits: orbits that after a finite amount of time come back to their starting point. Periodic orbits are thus (never selfintersecting) closed curves in Euclidean space, that is, they are knots. And yes, the Lorenz knots are exactly the periodic orbits of the Lorenz model.

It turns out that Lorenz knots fill out (they are dense, another typical feature of chaotic dynamical systems is the coexistence of periodic behavior with very wild behavior) the Lorenz attractor, and so understanding them might give important information on the structure of the Lorenz attractor. In the Eighties Joan Birman and Bob Williams [8] started studying Lorenz knots, trying to understand and classify them. They showed that all torus knots are Lorenz knots; and very recently Birman and Ilya Kofman have proved that every Lorenz knot is a twisted torus knot, a knot that can be obtained from a torus knot by a simple procedure (amounting to cutting the knot in several carefully chosen places, twisting the strands according to specific rules, and then gluing the strands together; see [9] for details).

\section{What is a modular knot?}

To explain what is a modular knot we must first explain what a lattice is.

Roughly speaking, a lattice is a discrete family of points (in a line, a plane, a space...) uniformly distributed. The easiest example of lattice is the set of integer numbers in the real line; and, in a sense, this is the only example of lattice in the line. Indeed, if we take any lattice in the line, up to a translation we can assume that it contains the origin; and up to a rescaling we can assume that it is normalized, that is that the distance between two consecutive points in the lattice is exactly one and thus we have recovered the integers. From a geometrical point of view, then, a lattice in the line is obtained by covering the line with infinitely many copies of the same basic block, an interval (of length one if the lattice is normalized). 
In the plane, the situation is considerably more complex. As building block for a lattice we can use a parallelogram; but even assuming (as we may up to a translation) that one of the vertices of the parallelogram is the origin, we still have infinitely many distinct cases to consider. If one vertex is the origin, to describe the parallelogram (and hence the lattice obtained by covering the plane with copies of the basic parallelogram) it suffices to give the coordinates of two other vertices, $v_{1}=$ $\left(a_{1}, b_{1}\right)$ and $v_{2}=\left(a_{2}, b_{2}\right)$. Furthermore, we can also assume that (up to a rescaling) the lattice is normalized, that is that the basic parallelogram has area one (conditions amounting to requiring that $a_{1} b_{2}-a_{2} b_{1}$ is equal to one).

So to describe a normalized lattice we need four real numbers (the coordinates of two vertices of the basic parallelogram) satisfying one condition (area equal to 1); this means that we can identify the space of all normalized lattices with a suitable subset of the Euclidean 3-space (actually one needs to add a point at infinity, getting a subset of the 3-dimensional sphere, but this is a detail). It turns out that this subset is exactly the complement of a trefoil knot - the first but not last appearance of knots in this setting.

There is another way of describing the space of normalized lattices. Instead of considering the two vertices separately, we can put their coordinates in a $2 \times 2$ matrix; the normalization condition then amounts to saying that the determinant of this matrix is 1 . If we multiply a matrix with determinant 1 by another matrix with determinant 1 we still get a matrix with determinant 1 , that is another normalized lattice. In particular, this holds if we multiply by the diagonal matrix having $e^{t}$ and $e^{-t}$ as diagonal elements, where $t$ is any real number. Letting $t$ vary in the real numbers, we then get a whole family of normalized lattices, that can be thought of as a curve in the complement of the trefoil knot, an orbit of the modular flow. See [10] for (a lot) more details.

The modular flow appears and is very important in several areas of number theory and onedimensional complex analysis; but the aspect that is interesting for us now is that the modular flow has periodic orbits, forming knots contained in the complement of the trefoil knot; these periodic orbits are (of course) called modular knots. It turns out that they are in one-to-one correspondence with (similarity classes of) $2 \times 2$ matrices with integer coefficients, determinant one and absolute value of the trace (the sum of the diagonal elements) greater than 2; these matrices are the hyperbolic elements of the modular group (the group of $2 \times 2$ matrices with integer coefficients and determinant 1). Notice that to give a modular knots it then suffices to give four integer numbers (satisfying a number of conditions); so it is not surprising that topological properties of modular knots have something to do with number theoretical properties of integer numbers.

Modular knots have been studied for a long time; however, Ghys found a new way of looking at them, giving unexpected results. 


\section{What do they have to do with each other?}

Ghys' surprising discovery is that a knot can be realized as a Lorenz knot if and only if it can be realized as a modular knot. In other words, the class of Lorenz knots coincide with the class of modular knots.

To prove this, Ghys gave a way to pass from a Lorenz knot to a modular knot and conversely, based on the idea of Lorenz template previously introduced by Birman and Williams. The Lorenz template (see again [2] and [3] for beautiful pictures) is a figure-eight-shaped surface, similar to and thus still sort of butterfly-like - but much simpler than the Lorenz attractor, with the very useful property that every Lorenz knot can be continuously pushed onto the Lorenz template (remaining equivalent to the original knot). Furthermore, the left wing and the right wing of the butterfly in the Lorenz template are joined by a central one-dimensional stick; and every Lorenz knot must cross this central stick. More precisely, Birman and Williams showed that a Lorenz knot is completely determined by the way it crosses the central stick, going into the left wing or the right wing after each crossing; the sequence of left/right choices is enough to completely reconstruct the given Lorenz knot.

What Ghys did was to find a (topologically equivalent) copy of the Lorenz template inside the space of normalized lattices (the complement of the trefoil knot), and to show how modular knots can be (following a natural geometric procedure) pushed down on this Lorenz template so to become Lorenz knots. Conversely, he also showed that every sequence of left/right choices at the central stick can be realized by a modular knot, and so all Lorenz knots are modular knots too.

This discovery has already had profound consequences in the theory of the modular flow (and thus in number theory and related areas). All properties of Lorenz knots must be enjoyed by modular knots, and conversely. For instance, modular knots must be fibered (that is, it should be possible to fill the complement of the knot by surfaces all having the boundary lying on the given knot, quite an unusual property for a knot to have) because (as Birman and Williams showed) all Lorenz knots are; at present a direct proof (that is a proof not using Lorenz knots) of this fact is not known.

Another unexpected consequence consists in a new way to compute the Rademacher function, a very useful number-theoretic object whose classical definition is very cumbersome, involving taking the complex logarithm of the $24^{\text {th }}$ root of something known as the Weierstrass discriminant, and then following the complex logarithm along a closed curve associated to a (hyperbolic) element of the modular group. Going along a closed curve the complex logarithm changes by an integer multiple of $2 \pi i$; this integer is the value of the Rademacher function computed in the given element 
of the modular group. Well, Ghys has shown that the Rademacher function is simply given by the number of the left choices minus the number of right choices made by the corresponding modular knot pressed onto the Lorenz template!

Ghys' discovery prompted new advances in the study of the Lorenz model too; for instance, the characterization of Lorenz (and hence modular) knots as twisted torus knots given by Birman and Kofman was inspired by Ghys' results. Furthermore, modular knots are much easier to generate than Lorenz knots, and since they still preserve all the topological features of Lorenz knots, in principle they might be used to explore the intricacies of the Lorenz attractor. In general, the appearance of important features of the Lorenz model in a completely different context seems to indicate that it was not a complete accident that the first chaotic system to be discovered was Lorenz'; possibly the Lorenz model is more basic, more intrinsic than we actually imagine.

This is probably just the beginning of a long and exciting story: new discoveries, new results and new unexpected connections might be waiting just around the corner. But even if this will not be the case, Ghys' work remains a beautiful piece of contemporary mathematics that will be studied and admired for a long time.

\section{References}

[1] GHYS, É.: Knots and dynamics. In International Congress of Mathematicians. Vol. I. European Mathematical Society, Zürich, 2007, pp. 247-277.

[2] http://www.josleys.com/articles/ams_article/Lorenz3.htm

[3] http://www.josleys.com/

[4] ADAMS, C.: The knot book. American Mathematical Society, Providence, RI, 2004.

[5] http://www.knotplot.com

[6] LORENZ, E.: Deterministic nonperiodic flow. J. Atmos. Sci. 20 (1963), 130-141

[7] http://www.cmp.caltech.edu/ mcc/Chaos_Course/Lesson1/Demo8.html

[8] BIRMAN, J. \& WILLIAMS, R.: Knotted periodic orbits in dynamical systems. I. Lorenz's equations. Topology 22 (1983), 47-82.

[9] BIRMAN, J. \& KOFMAN, I.: A new twist on Lorenz links. J. Topology 2 (2009), 227-248.

[10] SERRE, J.-P.: A course in arithmetic. Springer-Verlag New York, Heidelberg, 1973. 